\title{
Porosity Governs Normal Stresses in Polymer Gels
}

\author{
Henri C. G. de Cagny, ${ }^{1}$ Bart E. Vos, ${ }^{2}$ Mahsa Vahabi, ${ }^{3}$ Nicholas A. Kurniawan, ${ }^{2,4}$ Masao Doi, ${ }^{5}$ \\ Gijsje H. Koenderink, ${ }^{2, *}$ F. C. MacKintosh, ${ }^{3,6,7, \dagger}$ and Daniel Bonn ${ }^{1, \$}$ \\ ${ }^{1}$ Institute of Physics, University of Amsterdam, Science Park 904, 1098 XH Amsterdam, The Netherlands \\ ${ }^{2}$ FOM-Institute AMOLF, Science Park 104, 1098 XG Amsterdam, The Netherlands \\ ${ }^{3}$ Department of Physics and Astronomy, Vrije Universiteit, De Boelelaan 1081, 1081 HV Amsterdam, The Netherlands \\ ${ }^{4}$ Department of Biomedical Engineering and Institute for Complex Molecular Systems, \\ Eindhoven University of Technology, 5612 AP Eindhoven, The Netherlands \\ ${ }^{5}$ Center of Soft Matter Physics and its Applications, Beihang University, 100191 Beijing, China \\ ${ }^{6}$ Departments of Chemical \& Biomolecular Engineering, Chemistry, and Physics \& Astronomy, \\ Rice University, Houston, 77005 Texas, USA \\ ${ }^{7}$ Center for Theoretical Biophysics, Rice University, Houston, 77030 Texas, USA
}

(Received 11 June 2016; revised manuscript received 22 September 2016; published 18 November 2016)

\begin{abstract}
When sheared, most elastic solids including metals, rubbers, and polymer gels dilate perpendicularly to the shear plane. This behavior, known as the Poynting effect, is characterized by a positive normal stress. Surprisingly, fibrous biopolymer gels exhibit a negative normal stress under shear. Here we show that this anomalous behavior originates from the open-network structure of biopolymer gels. Using fibrin networks with a controllable pore size as a model system, we show that the normal-stress response to an applied shear is positive at short times, but decreases to negative values with a characteristic time scale set by pore size. Using a two-fluid model, we develop a quantitative theory that unifies the opposite behaviors encountered in synthetic and biopolymer gels.
\end{abstract}

DOI: 10.1103/PhysRevLett.117.217802

When subjected to a shear stress, materials either shrink (shear contraction) or expand (shear dilatancy). As shown by Poynting more than a century ago [1], simple elastic solids exhibit shear dilatancy. Similar behavior has since been observed in more complex viscoelastic systems, such as granular materials, rubbers, and polymer glasses $[2,3]$. The tendency of such materials to dilate can be measured as a positive normal compressive stress or pressure that develops when a sample is sheared between two plates with a fixed gap. In case of polymer materials, shear dilatancy is usually described by the classical Mooney-Rivlin model [4,5], which predicts a normal-stress difference $N_{1} \sim G \gamma^{2}$, where $\gamma$ is the shear strain and $G$ the network shear modulus. In Fig. 1(a) this behavior is illustrated for polyacrylamide (PAAm) hydrogels of varying stiffness subjected to an oscillatory shear deformation. Surprisingly, biopolymer networks have been reported to exhibit the opposite response, contracting when sheared [6-8]. This behavior is clearly illustrated in Fig. 1(b), which shows that aqueous gels of the bloodclotting protein fibrin develop a negative normal stress under shear. The magnitude of the normal stress again increases quadratically with strain, but it reaches values comparable to the shear modulus at significantly lower shear strain $(\gamma \simeq 1 / 10)$ than for polyacrylamide $(\gamma \simeq 1)$. The origin for the remarkable difference in the sign and magnitude of the normal stress between synthetic hydrogels and biopolymer gels is still unknown.

Here, we aim to understand the mechanistic basis of the fundamentally different response of synthetic and biopolymer gels and to develop a minimal model that can capture the behavior of both types of gels. In either case, the normal-stress response is fundamentally nonlinear, since its sign cannot reverse when the shear train $\gamma$ is reversed. Thus, to lowest order, normal stress is expected to vary as $\gamma^{2}$, even while the shear stress remains linear in $\gamma$. Although both gels in Fig. 1 are hydrogels containing over 90\% interstitial water, there is structurally a profound difference in the pore size. While polyacrylamide gels have a pore size of order $10 \mathrm{~nm}$ [16], fibrin networks have pore sizes that can be in the micrometer range [17-19]. Fluid permeability can therefore play an important role in the mechanical response. For hydrogels with a small pore size, we expect a strong viscous coupling between the network and the solvent, which will suppress motion of the network relative to the solvent and effectively render the gel, as a whole, incompressible. By contrast, biopolymer gels can expel interstitial fluid to relax pressure gradients on long-enough time scales, allowing the network to contract upon shearing [7,20-22].

To study the role of porosity, we choose fibrin networks as a model system, the pore size of which can be tuned from nanometers to micrometers by simply changing the temperature, ionic strength, and $p \mathrm{H}$ during self-assembly [23]. This is demonstrated in Figs. 2(a) and 2(b), which show fluorescence microscopy images of two fibrin gels that are assembled at the same monomer concentration of $8 \mathrm{mg} / \mathrm{mL}$ but at different temperatures. Using quantitative measurements of the fiber mass-length ratio by light 


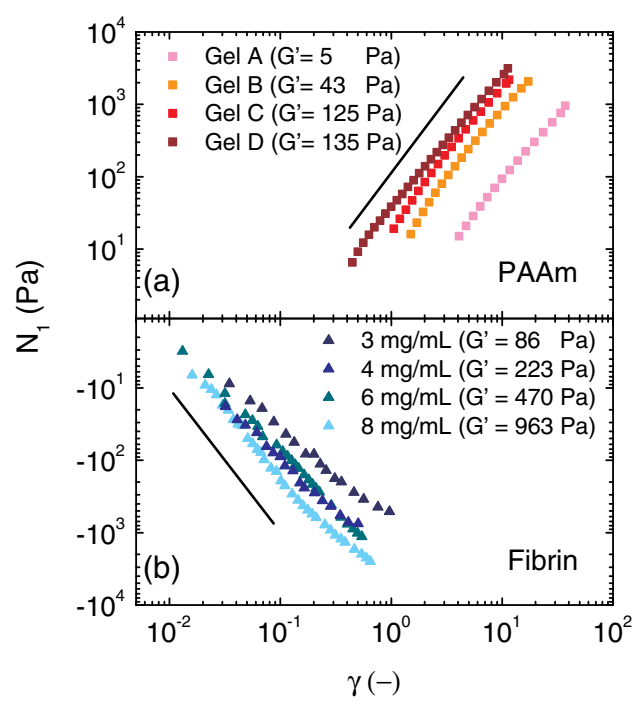

FIG. 1. Normal stress difference $N_{1}=2 F / \pi R^{2}$, where $F$ is the normal force (thrust) reported by the rheometer and $R$ is the sample radius, as a function of the amplitude of the applied oscillatory shear strain. (a) $N_{1}$ for PAAm [9] prepared with various ratios of monomer-to-cross-linker concentrations. The line indicates a quadratic dependence of $N_{1} \sim \gamma^{2}$, as expected from the Mooney-Rivlin model [4,5]. (b) $N_{1}$ shown for fibrin gels polymerized at $22^{\circ} \mathrm{C}$ at various fibrinogen concentrations (in $\mathrm{mg} / \mathrm{mL}$ ). The line indicates a $\sim \gamma^{2}$ dependence, but with negative sign.

scattering, we calculate average mesh sizes of 0.36 and $0.29 \mu \mathrm{m}$ for these networks (see [9] for further details).

To test the influence of pore size on the normal-stress response, we subject each network to a constant shear stress and we monitor the normal stress as a function of time. Intriguingly, we find that in each case, the normal stress relaxes from an initially positive or close-to-zero value to a negative steady-state value with a rate that strongly varies with pore size. The characteristic relaxation time, $\tau$, increases from just a few seconds to $\sim 100 \mathrm{~s}$ as the pore size of fibrin decreases from 0.36 to $0.08 \mu \mathrm{m}$. For PAAm gels with a pore size $\sim 10 \mathrm{~nm}$ [16], the relaxation time grows to over 15 hours [Fig. 2(c)]. These observations support our hypothesis that the sign of the normal stress is controlled by the time scale for solvent flow through the network. The data suggest that the normal stress is positive as long as the polymer network and the fluid remain viscously coupled, and switches sign to become negative when the fluid can move relative to the network. Importantly, the time scale separating these behaviors is unrelated to the time scales apparent in the linear viscoelastic response [see Fig. 2(c)].

To quantitatively model the effects of network poroelasticity in the shear rheology of polymer networks, we start from the two-fluid model [24-27] that describes a polymer gel as a biphasic system composed of a linear elastic network immersed in a viscous and incompressible liquid [9]. The two components are coupled by a force per unit volume, $\Gamma(\dot{\vec{u}}-\vec{v})$, acting on the liquid and opposite

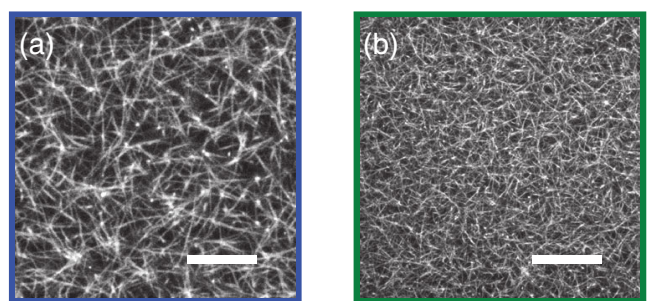

(c)

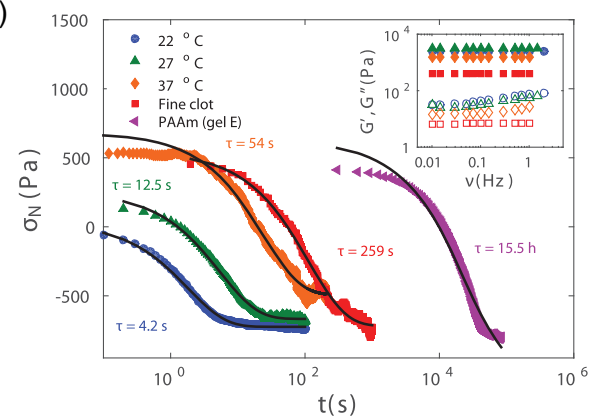

(d)

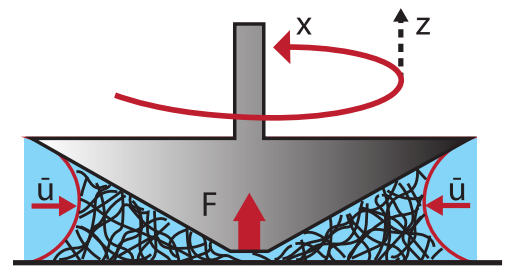

FIG. 2. (a),(b) Fluorescence confocal microscopy images of fibrin networks whose pore size is tuned by polymerizing under different conditions, at (a) $22^{\circ} \mathrm{C}$ and (b) $27^{\circ} \mathrm{C}$. The scale bars are $10 \mu \mathrm{m}$. Protein content is $(8 \mathrm{mg} / \mathrm{mL})$ in both samples. (c) Normal stress $\sigma_{N}$, given by the apparent normal-stress difference $2 F / \pi R^{2}$ obtained from the rheometer thrust $F$, for four fibrin networks differing in pore size as a function of time after the application of a constant shear stress at $t=0$. The stress relaxation curves are fitted to an exponential decay derived from the two-fluid model in [9] (black lines). The viscoelastic time scale is unrelated to the normal-stress transition, as shown in the inset where the storage moduli (filled symbols) and the loss moduli (open symbols) of the gels are plotted. (d) Schematic representation of the two-fluid model showing an inward, radial contraction of the network (black) relative to the solvent (blue) upon shearing.

to the force on the network. This dissipative force arises from the relative motion of the solvent, which moves with velocity $\vec{v}$ and the network, with velocity $\dot{\vec{u}}$. For a network with pore size $\xi$ and a fluid with viscosity $\eta, \Gamma \sim \eta / \xi^{2}$, since the Stokes drag force on a network strand of size $\sim \xi$ moving with relative velocity $\sim v$ is $\sim \eta \xi \Delta v$ and this acts on a volume $\sim \xi^{3}$ [9]. Given the small polymer volume fraction $\phi$ of most hydrogels and biopolymer networks ( $\phi \sim 10^{-3}$ for fibrin gels), the radial velocity component of the incompressible fluid effectively vanishes and the only radial motion is due to the network. This radial motion, $\dot{u}_{r}$, generates a radial pressure gradient in the solvent given by

$$
\nabla_{r} P=\Gamma \dot{u}_{r}=-\tilde{\sigma} / r-\left(K / r^{2}\right) u_{r},
$$


for a cone-plate geometry. The net force on the network has two distinct elastic contributions. The first contribution comes from the hoop stress $\tilde{\sigma}$, which tends to drive the network radially inward [Fig. 2(d)] [9]: hoop stresses generated by shearing tend to drive radial contraction of the network and expulsion of the solvent, much as a twisted sponge expels water by contracting radially. By symmetry, $\tilde{\sigma} \sim \gamma^{2}$ to lowest order, as noted above, although Eq. (1) is linear in $u_{r}$. The second contribution to the net force on the network comes from a restoring force that balances the radial contraction on long time scales (i.e., as $\dot{u}_{r} \rightarrow 0$ ). This restoring force originates from the gradient in the elastic shear stress $\sim G \nabla_{z} u_{r}$ that results from the axial (z) variation of $u$ [see Fig. 2(d)]. For a cone-plate rheometer with small gap size $d$ and small cone angle $\alpha$, the restoring force $\sim G / d^{2} u_{r}$. Thus, since $d=\tan (\alpha) r, K \sim G / \tan (\alpha)^{2}$ in Eq. (1). We thus predict a characteristic relaxation time $\tau \sim \eta d^{2} / G \xi^{2}$. Indeed, we experimentally observe a rapid decrease of the relaxation time with increasing pore size, consistent with the predicted scaling (see Fig. S1 in [9]).

We can consider two opposite limits of Eq. (1). In the limit of small pore size and $\Gamma \rightarrow \infty$, the radial displacement $u_{r} \rightarrow 0$ (with finite $\Gamma \dot{u}_{r}$ ) and Eq. (1) reduces to $\nabla_{r} P=-\tilde{\sigma} / r$. Shearing will thus increase the pressure toward the axis of the rheometer, which results in a positive contribution to the normal force. Dense hydrogels will therefore effectively behave as incompressible materials for which the normal force $F$ is related to the normal-stress difference $\sigma_{x x}-\sigma_{z z}$ by $N_{1}=2 F / \pi R^{2}$, where $R$ is the sample radius [28]. $N_{1}$ is positive for a rubberlike material, consistent with measurements on polyacrylamide gels [5]. In the opposite limit of networks with a large pore size, the pressure difference can relax by water efflux and in steady state the two terms on the right-hand side of Eq. (1) cancel. In the absence of this pressure, only the polymer stress terms remain and the normal force measured by the rheometer is given by $F=-\pi R^{2} \sigma_{z z}$, corresponding to a reported (apparent) normal-stress difference $N_{1}^{(\text {app })}=-2 \sigma_{z z}$.

A key prediction of the two-fluid model is that the response of the normal stress measured in a rheology experiment should depend on the experimental time scale relative to the characteristic relaxation time, $\tau \sim \eta d^{2} / G \xi^{2}$. To quantitatively test this prediction, we subject the fibrin gels to an oscillatory shear stress with frequencies between 0.001 and $5 \mathrm{~Hz}$, allowing us to conveniently probe a range of time scales from 0.2 to $1000 \mathrm{~s}$ in a single experiment. We measure the normal stress response after the system has reached steady state (Fig. S2 in [9]). We focus on fibrin gels polymerized at $27^{\circ} \mathrm{C}$, which have a relaxation time $\tau \approx$ $12.5 \mathrm{~s}$ that lies in the middle of the experimentally accessible frequency range. In steady state, the observed normal stresses are indeed negative over the entire frequency range.

When we plot the time-dependent normal stress [Figs. 3(d)-(f)] as a function of shear stress, we obtain the Lissajous curves shown in Figs. 3(a)-3(c). Strikingly,
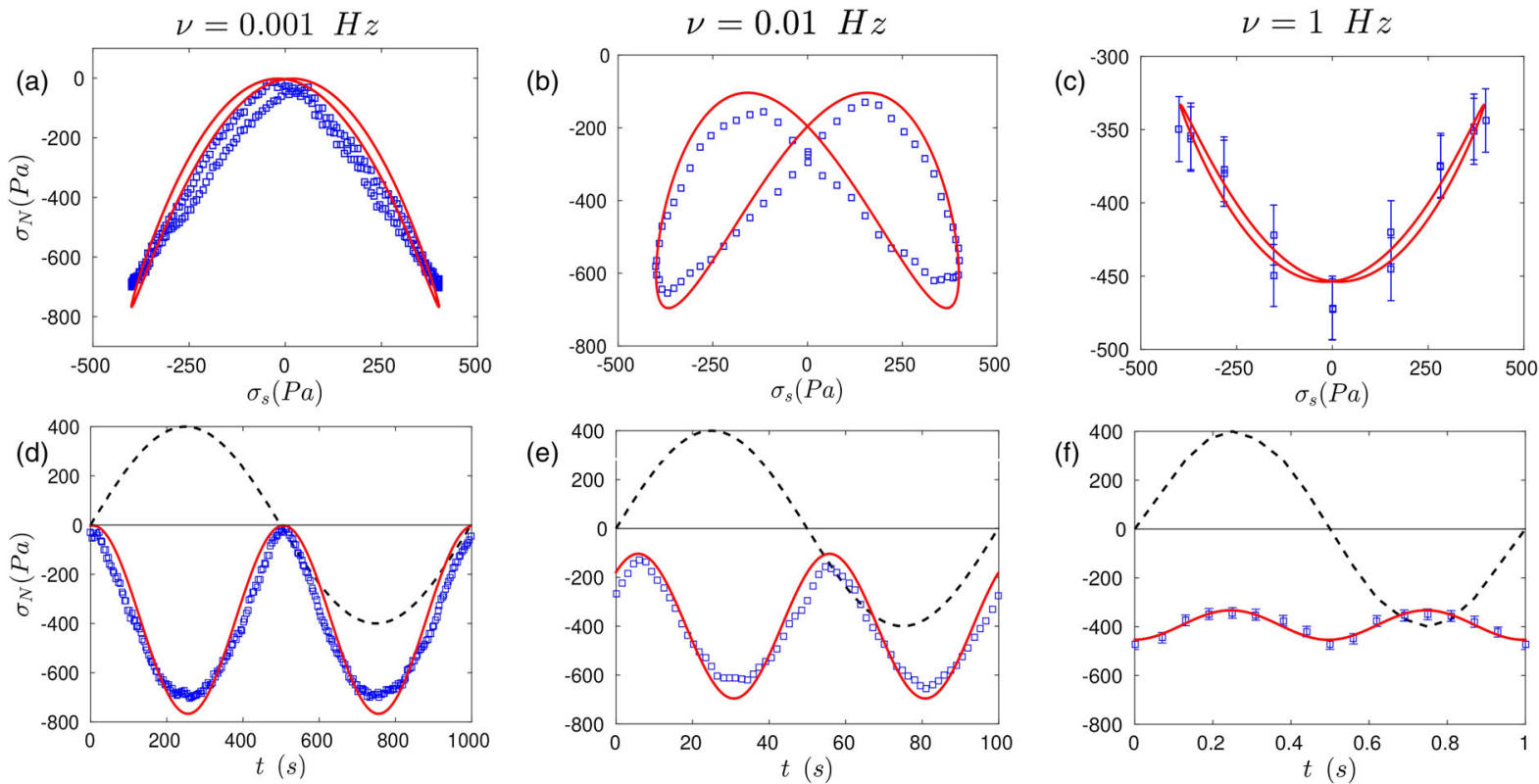

(e)

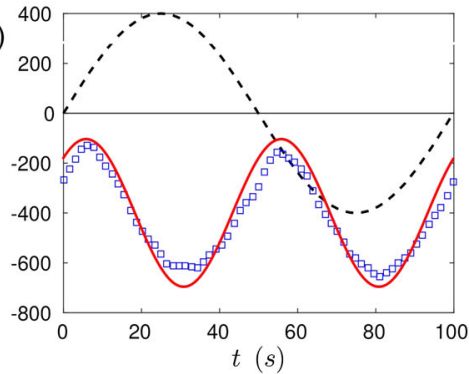

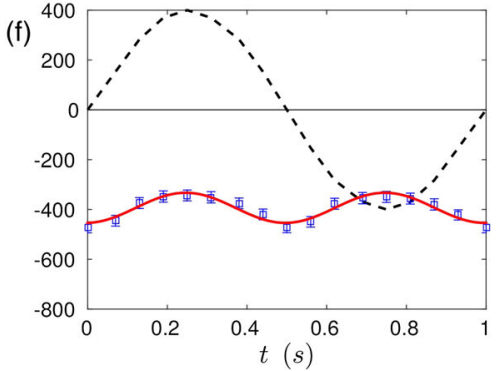

FIG. 3. Normal stress $\sigma_{N}$, given by the apparent normal stress difference $N_{1}^{(\text {app })}=2 F / \pi R^{2}$ reported by the rheometer, for a fibrin gel polymerized at $27^{\circ} \mathrm{C}$ in response to an oscillatory shear stress at oscillation frequencies of $0.001 \mathrm{~Hz}$ (left), $0.01 \mathrm{~Hz}$ (middle), and $1 \mathrm{~Hz}$ (right). The top panels [(a)-(c)] show the Lissajous curves of normal stress versus shear stress (symbols) fitted by the predictions of the two-fluid model in Eq. 2 (red lines) assuming a time constant of $12.5 \mathrm{~s}$. The bottom panels [(d)-(f)] show the corresponding timedependent normal stress (blue open symbols) and applied shear stress (black dotted lines). The data shown at $\nu=1 \mathrm{~Hz}$ represent averages with standard deviations obtained by averaging over 34 cycles to compensate for the low sampling frequency of the rheometer. The normal stresses are all negative since they correspond to steady-state values, obtained after initial relaxation (Fig. S2 in [9]). 
the Lissajous curves completely change with changing frequency. For oscillation periods longer than $\tau$ [Fig. 3(a)], the normal stress decreases with increasing shear stress, demonstrating contractile behavior under shear. By contrast, for oscillation periods shorter than $\tau$, the normal stress increases with increasing shear stress, demonstrating extensile behavior [Fig. 3(c)]. The transition occurs at an intermediate frequency that is of order $1 / \tau$ [Fig. 3(b)]. This experiment unambiguously shows that the normal stress response of a polymer gel is governed by fluid flow, which is suppressed at higher frequencies. The normal stress response is therefore controlled by the network pore size, and is furthermore dependent on the shear modulus $G$ and the gap size $d$ between the cone and the plate (see Fig. S3 in [9]).

The two-fluid model allows us to perform an even more rigorous test of the mechanism governing the normal stress response of polymer gels, since we can calculate the time dependence of the normal stress and compare it to experiments. For symmetry reasons, the normal-stress components $\sigma_{x x}$ and $\sigma_{z z}$ are expected to have a leading $\gamma^{2}$ dependence on strain. Since the shear stress $\sigma_{x z} \simeq G \gamma$, we define $\sigma_{x x} \equiv A_{x} G \gamma^{2}$ and $\sigma_{z z} \equiv A_{z} G \gamma^{2}$. For an oscillatory strain $\gamma(t)=\gamma_{0} \sin (\omega t)$, the steady-state solution of the time-dependent Eq. (1) is [9]

$N_{1}^{(\mathrm{app})}=-2 A_{z} G \gamma(t)^{2}+\tilde{A} G \gamma_{0}^{2}[\mathcal{A} \cos (2 \omega t)+\mathcal{B} \sin (2 \omega t)]$,

where

$$
\begin{gathered}
\mathcal{A}=-\frac{1}{8 \omega \tau}\left[2 \tan ^{-1}(1+2 \sqrt{\omega \tau})\right. \\
\left.+2 \tan ^{-1}(1-2 \sqrt{\omega \tau})-\pi+4 \omega \tau\right], \\
\mathcal{B}=\frac{1}{8 \omega \tau} \log \left(1+4 \omega^{2} \tau^{2}\right),
\end{gathered}
$$

with $\tilde{A}=A_{x}-A_{z}$ and $\tau=\Gamma R^{2} / K \sim \eta d^{2} / G \xi^{2}$. In the limit where $\omega \tau \gg 1$, Eq. (2) reduces to the well-known MooneyRivlin expression for incompressible materials, $N_{1}=G \gamma^{2}$. In the opposite limit where $\omega \tau \ll 1$, Eq. (2) instead reduces to $N_{1}^{(\text {app })}=-2 A_{z} G \gamma^{2}$.

Based on prior measurements on a range of biopolymer gels in the $\omega \tau \ll 1$ limit $[6,7,29]$ as well as models of fibrous networks $[20,21,29,30]$, we anticipate $A_{z} \sim 1 / \gamma_{c}$, where $\gamma_{c}$ is the onset strain for nonlinear elasticity, which is typically $\sim 1 / 10$. Thus, in the limit of low frequencies, not only is $N_{1}^{(\text {app })}$ negative, but its magnitude can actually be much larger than $\sigma_{x y} \gamma$.

To test these predictions experimentally, we fit the oscillatory normal-stress data shown in Fig. 3 to Eq. (2). The only fit parameters are $A_{z}$ and $\tilde{A}$, since the shear modulus $G$ is measured independently from the shear stress at small strain and the relaxation time $\tau$ is measured independently from the normal-stress relaxation upon applying a constant shear stress $[\tau=12.5 \mathrm{~s}$, Fig. 2(c)]. We observe excellent agreement between the data (symbols) and the model (solid lines) over the entire range of oscillation frequencies (Fig. 3), with fitting parameters that are insensitive to frequency (Fig. S1 in [9]).

Our observations reveal that poroelastic effects involving interstitial fluid flow play an unexpectedly important role in the shear rheology of polymer gels. Poroelastic effects in porous media such as fluid-imbibed polymer gels are usually considered to affect only volume-changing deformations such as compression and extension [31-33]. Our experiments and theory demonstrate that the shear response of polymer gels is highly sensitive to fluid flow and network compressibility, in spite of the volume-conserving nature of simple shear deformations. Depending on the time scale of deformation and the hydrodynamic coupling of the polymer network with the surrounding solvent, polymer gels behave as either incompressible materials with a positive normal stress or compressible materials with a negative normal stress.

We demonstrated that the normal-stress response of both synthetic and biopolymer gels is quantitatively captured by a minimal model that takes into account the biphasic nature of hydrogels. This model can explain why synthetic hydrogels exhibit shear dilation, while biopolymer gels have been reported to exhibit shear contraction. This suggests a new route to tailor the sign and magnitude of the normal stresses for polymer materials by tuning the pore size, solvent viscosity, and nonlinear shear elasticity. This could prove valuable in the context of materials science, since normal stresses can cause elastic instabilities that severely complicate processing [3]. Finally, our findings highlight the important role of poroelastic effects in tissue and extracellular matrix mechanics, where normal stresses can become a dominant stress component, even for small strains of order 10\% [7]. Related poroelastic effects in intracellular networks have previously been shown to govern the rheology of cells. However, the much-smaller cellular dimensions $d \simeq 1 \mu \mathrm{m}$ can be expected to limit the corresponding poroelastic relaxation time to be of order $1 \mathrm{~s}$, even for the smaller mesh sizes of order $10 \mathrm{~nm}$ [34], which renders cells effectively compressible on time scales $\gtrsim 1 \mathrm{~s}$.

This work was supported by the Foundation for Fundamental Research on Matter (FOM), which is part of the Netherlands Organisation for Scientific Research (NWO).

H. C. G. d. C., B. E. V., and M. V. contributed equally to this work.

\footnotetext{
*g.koenderink@amolf.nl

fcmack@gmail.com

\$d.bonn@uva.nl
}

[1] J. H. Poynting, Proc. R. Soc. A 82, 546 (1909). 
[2] O. Reynolds, Philos. Mag. 20, 469 (1885).

[3] R. G. Larson, The Structure and Rheology of Complex Fluids (Oxford University Press, New York, 1998), p. 688.

[4] M. Mooney, J. Appl. Phys. 11, 582 (1940).

[5] R. S. Rivlin, Phil. Trans. R. Soc. A 241, 379 (1948).

[6] P. A. Janmey, M. E. McCormick, S. Rammensee, J. L. Leight, P. C. Georges, and F. C. MacKintosh, Nat. Mater. 6, 48 (2007).

[7] H. Kang, Q. Wen, P. A. Janmey, J. X. Tang, E. Conti, and F. C. MacKintosh, J. Phys. Chem. B 113, 3799 (2009).

[8] C. O. Horgan and J. G. Murphy, J. Elast. 118, 127 (2015).

[9] See Supplemental Material at http://link.aps.org/ supplemental/10.1103/PhysRevLett.117.217802, which includes [10-15], for Materials and Methods and a further note on the two-fluid model and relaxation of hoop stresses.

[10] M. D. Bale, M. F. Müller, and J. D. Ferry, Proc. Natl. Acad. Sci. U.S.A. 82, 1410 (1985).

[11] C. Yeromonahos, B. Polack, and F. Caton, Biophys. J. 99, 2018 (2010).

[12] I. K. Piechocka, K. A. Jansen, C. P. Broedersz, N. A. Kurniawan, F. C. MacKintosh, and G. H. Koenderink, Soft Matter 12, 2145 (2016).

[13] S. Münster and B. Fabry, Biophys. J. 104, 2774 (2013).

[14] J. N. Kapur, P. K. Sahoo, and A. K. C. Wong, Comput. Vis. Graph Image Process 29, 273 (1985).

[15] N. Otsu, IEEE Trans. Syst. Man Cybern. 9, 62 (1979).

[16] M. Tombs, Anal. Biochem. 13, 121 (1965).

[17] M. Okada, B. Blomback, M. D. Chang, and B. Horowitz, J. Biol. Chem. 260, 1811 (1985).

[18] M. Pieters, A. Undas, R. Marchi, M. P. M. De Maat, J. W. Weisel, and R. A. S. Ariëns, J. Thromb. Haemostasis 10, 2179 (2012).
[19] N. R. Lang, S. Münster, C. Metzner, P. Krauss, S. Schürmann, J. Lange, K. E. Aifantis, O. Friedrich, and B. Fabry, Biophys. J. 105, 1967 (2013).

[20] C. Heussinger, B. Schaefer, and E. Frey, Phys. Rev. E 76, 031906 (2007).

[21] E. Conti and F. C. MacKintosh, Phys. Rev. Lett. 102, 088102 (2009).

[22] B. P. Tighe, Granular Matter 16, 203 (2014).

[23] C. Nunes and M. Roedersheimer, Microgravity Sci. Technol. 8, 125 (1995).

[24] F. Brochard and P. G. de Gennes, Macromolecules 10, 1157 (1977).

[25] S. T. Milner, Phys. Rev. E 48, 3674 (1993).

[26] F. Gittes, B. Schnurr, P. D. Olmsted, F. C. MacKintosh, and C. F. Schmidt, Phys. Rev. Lett. 79, 3286 (1997).

[27] A. J. Levine and T. C. Lubensky, Phys. Rev. Lett. 85, 1774 (2000).

[28] D. C. Venerus, Appl. Rheol. 17, 1 (2007).

[29] C. Storm, J. J. Pastore, F. C. MacKintosh, T. C. Lubensky, and P. A. Janmey, Nature (London) 435, 191 (2005).

[30] F. C. MacKintosh, J. Käs, and P. A. Janmey, Phys. Rev. Lett. 75, 4425 (1995).

[31] M. A. Biot, J. Appl. Phys. 27, 459 (1956).

[32] L. Casares, R. Vincent, D. Zalvidea, N. Campillo, D. Navajas, M. Arroyo, and X. Trepat, Nat. Mater. 14, 343 (2015).

[33] A. S. G. van Oosten, M. Vahabi, A. J. Licup, A. Sharma, P. A. Galie, F. C. MacKintosh, and P. A. Janmey, Sci. Rep. 6, 19270 (2016).

[34] E. Moeendarbary, L. Valon, M. Fritzsche, A. R. Harris, D. A. Moulding, A. J. Thrasher, E. Stride, L. Mahadevan, and G. T. Charras, Nat. Mater. 12, 253 (2013). 